Author affiliations appear at the end of this article.

Published online ahead of print at www.jco.org on January 20, 2015

Written on behalf of the International Myeloma Working Group.

Terms in blue are defined in the glossary, found at the end of this article and online at www.jco.org.

Authors' disclosures of potential conflicts of interest are found in the article online at www.jco.org. Author contributions are found at the end of this article.

Corresponding author: Meletios A Dimopoulos, MD, Department of Clinical Therapeutics, National and Kapodistrian University of Athens School of Medicine, Alexandra General Hospital, 80 Vas. Sofias Ave, 11528, Athens,

Greece; e-mail: mdimop@med.uoa.gr.

(C) 2015 by American Society of Clinical Oncology

0732-183X/15/3306w-657w/\$20.00 DOI: 10.1200/JCO.2014.57.996

\title{
Role of Magnetic Resonance Imaging in the Management of Patients With Multiple Myeloma: A Consensus Statement
}

Meletios A. Dimopoulos, Jens Hillengass, Saad Usmani, Elena Zamagni, Suzanne Lentzsch, Faith E. Davies, Noopur Raje, Orhan Sezer, Sonja Zweegman, Jatin Shah, Ashraf Badros, Kazuyuki Shimizu, Philippe Moreau, Chor-Sang Chim, Juan José Lahuerta, Jian Hou, Artur Jurczyszyn, Hartmut Goldschmidt, Pieter Sonneveld, Antonio Palumbo, Heinz Ludwig, Michele Cavo, Bart Barlogie, Kenneth Anderson, G. David Roodman, S. Vincent Rajkumar, Brian G.M. Durie, and Evangelos Terpos

See accompanying editorial on page 537

$$
\begin{array}{llllllll}
\text { A } & \text { B } & \mathbf{S} & \mathbf{T} & \mathbf{R} & \mathbf{A} & \mathbf{C} & \mathbf{T}
\end{array}
$$

\section{Purpose}

The aim of International Myeloma Working Group was to develop practical recommendations for the use of magnetic resonance imaging (MRI) in multiple myeloma (MM).

\section{Methods}

An interdisciplinary panel of clinical experts on $\mathrm{MM}$ and myeloma bone disease developed recommendations for the value of MRI based on data published through March 2014.

\section{Recommendations}

MRI has high sensitivity for the early detection of marrow infiltration by myeloma cells compared with other radiographic methods. Thus, MRI detects bone involvement in patients with myeloma much earlier than the myeloma-related bone destruction, with no radiation exposure. It is the gold standard for the imaging of axial skeleton, for the evaluation of painful lesions, and for distinguishing benign versus malignant osteoporotic vertebral fractures. MRI has the ability to detect spinal cord or nerve compression and presence of soft tissue masses, and it is recommended for the workup of solitary bone plasmacytoma. Regarding smoldering or asymptomatic myeloma, all patients should undergo whole-body MRI (WB-MRI; or spine and pelvic MRI if WB-MRI is not available), and if they have > one focal lesion of a diameter $>5 \mathrm{~mm}$, they should be considered to have symptomatic disease that requires therapy. In cases of equivocal small lesions, a second MRI should be performed after 3 to 6 months, and if there is progression on $\mathrm{MRI}$, the patient should be treated as having symptomatic myeloma. $\mathrm{MRI}$ at diagnosis of symptomatic patients and after treatment (mainly after autologous stem-cell transplantation) provides prognostic information; however, to date, this does not change treatment selection.

\section{J Clin Oncol 33:657-664. (C) 2015 by American Society of Clinical Oncology}

\section{INTRODUCTION}

Bone disease, characterized by the presence of osteolytic lesions, bone fractures, or osteoporosis, is a significant cause of morbidity and mortality in multiple myeloma (MM). Therefore, the guidelines of the International Myeloma Working Group (IMWG) suggest that the presence of even asymptomatic bone disease on conventional radiography is a criterion of symptomatic MM that requires treatment. ${ }^{1}$

In 2009, the IMWG indicated that whole-body (WB) $\mathrm{x}$-ray (WBXR) remains the gold standard for the evaluation of MM-related bone disease. ${ }^{2}$ However, the detection limit of WBXR is low; to detect an osteolytic lesion by WBXR, a proportion of at least $30 \%$ to $50 \%$ of the trabecular bone has to be resorbed. ${ }^{3}$ Moreover, WBXR is not a suitable technique for the diagnosis of myeloma-related osteoporosis, has low visualization of the spine and pelvis, and cannot accurately depict the cause of painful lesions in patients with MM. In previous recommendations, the IMWG supported the implementation of magnetic resonance imaging (MRI) in the absence of osteolytic lesions on WBXR. ${ }^{2}$ However, the IMWG did not suggest the use of MRI for the definition of symptomatic myeloma. Thus, to date, a patient with focal lesions on MRI but with no lytic lesions on WBXR and with no other CRAB (hypercalcemia, renal failure, anemia, and bone disease) 


\begin{tabular}{|c|c|}
\hline Rating & Description \\
\hline \multicolumn{2}{|l|}{ Level } \\
\hline I & $\begin{array}{l}\text { Evidence obtained from meta-analysis of multiple well-designed, } \\
\text { controlled studies; randomized trials with low false-positive } \\
\text { and low false-negative errors (high power) }\end{array}$ \\
\hline$\|$ & $\begin{array}{l}\text { Evidence obtained from at least one well-designed experimental } \\
\text { study; randomized trials with high false-positive and/or false- } \\
\text { negative errors (low power) }\end{array}$ \\
\hline III & $\begin{array}{l}\text { Evidence obtained from well-designed, quasiexperimental } \\
\text { studies (eg, nonrandomized, controlled single-group, pre-post, } \\
\text { cohort, time, or matched case-control studies) }\end{array}$ \\
\hline IV & $\begin{array}{l}\text { Evidence from well-designed, nonexperimental studies (eg, } \\
\text { comparative and correlational descriptive and case studies) }\end{array}$ \\
\hline V & Evidence from case reports and clinical examples \\
\hline \multicolumn{2}{|l|}{ Grade } \\
\hline A & $\begin{array}{l}\text { Evidence of type I or consistent findings from multiple studies } \\
\text { of types II, III, or IV }\end{array}$ \\
\hline B & Evidence of type II, III, or IV; findings are generally consistent \\
\hline C & Evidence of type II, III, or IV; findings are inconsistent \\
\hline D & Little or no systematic empiric evidence \\
\hline
\end{tabular}

criteria is considered to have smoldering or asymptomatic myeloma (SMM), and follow-up with no treatment is recommended. Several novel data stress the value of MRI in this setting, and we suggest that the current treatment practice be changed for these patients. Our aim was to produce useful recommendations for the use of MRI in everyday clinical practice for the management of patients with myeloma and introduce novel MRI criteria for the definition of SMM.

\section{METHODS}

An interdisciplinary panel of experts on myeloma bone disease and MRI performance in patients with myeloma developed the recommendations based on evidence of published clinical or observational studies, metaanalyses, and systematic reviews through March 2014. Expert consensus was used to propose recommendations in the absence of sufficiently published data. Levels of evidence and grades of recommendations were used according to established criteria (Table 1). The statement was drafted and circulated among all panel members, followed by subsequent rounds of revision until consensus was achieved.

\section{MRI TECHNIOUES FOR MYELOMA}

Several MRI techniques have been developed for the assessment of bone marrow involvement in MM: T1 weighted, T2 weighted with fat suppression, short time inversion recovery, and gadolinium $\mathrm{T} 1$ weighted with fat suppression. ${ }^{4}$ Myeloma lesions typically show a low signal intensity on T1-weighted images, a high signal intensity on T2-weighted and short time inversion recovery images, and often enhancement on gadolinium-enhanced images. ${ }^{5,6}$ Table 2 summarizes the minimum technical requirements of these techniques for the management of patients with myeloma.

Limitations of MRI are the prolonged acquisition time, high cost, exclusion of patients with metal devices in their bodies, difficulties in cases of claustrophobic patients, and limited field of view. To override these restrictions, a WB-MRI methodology, which does not usually require contrast infusion, was developed. The time of WB-MRI is
Table 2. Minimum Technical Requirements for Use of MRI in Patients With Myeloma

\begin{tabular}{ll}
\hline \multicolumn{1}{c}{ Type } & \multicolumn{1}{c}{ Requirement } \\
\hline $\begin{array}{l}\text { Coil } \\
\begin{array}{c}\text { Examination } \\
\text { volume }\end{array}\end{array}$ & Target volume adapted surface-reception coil system \\
$\begin{array}{l}\text { Orientation } \\
\text { Parameter }\end{array}$ & Sagittal axial for spine; axial coronal for pelvis \\
Slice thickness & $4 \mathrm{~mm}$ (definitely not $>5 \mathrm{~mm}$ ) \\
Matrix & 512 pixels \\
Weighting & T1, T2 \\
Sequences & Sagittal T1 TSE, sagittal or coronal TIRM (STIR), axial T1 \\
& and T2 TSE, chemical shift, and T1 post contrast \\
\hline
\end{tabular}

Abbreviations: MRI, magnetic resonance imaging; STIR, short time inversion recovery; TIRM, turbo inversion recovery magnitude; TSE, turbo spin echo; WB, whole body.

approximately 45 minutes. Although of interest, this newer technique is not yet widely employed.

The MRI methods mentioned use MRI exquisite contrast and spatial resolution for the depiction of WB anatomy and specific tissue composition in detail. A newer MRI sequence is diffusionweighted imaging (DWI). This functional technique demonstrates alterations in intra- and extracellular water content from disruption of the transmembrane water flux that are visible before identified changes on the morphologic routine sequences. ${ }^{7-9}$ One disadvantage of DWI is that the apparent diffusion coefficient (ADC) is not exclusively influenced by diffusion but also by perfusion. However, improved sequences are under development to differentiate both influences. ${ }^{10}$ DWI can be used to detect regions with bone marrow infiltration for both diagnosis and monitoring of treatment response. ${ }^{1,12}$ In patients with MM, the ADC was reproducible $^{13}$ and correlated with bone marrow cellularity and microvessel density (MVD). ${ }^{14}$

The dynamic contrast-enhanced MRI (DCE-MRI) is another MRI technique in which the distribution of a contrast agent inside and outside the blood vessels is assessed by computer-based analysis of repeated images over time. The analysis provides data for blood volume and vessel permeability for the assessment of microcirculation. ${ }^{15,16}$ More importantly, in patients with MM, DCE-MRI measurements have been correlated with marrow angiogenesis and $\mathrm{MVD}^{17}$ as well as with angiogenic response to therapy. ${ }^{18}$ Regarding DCE-MRI sampling rate and model, there are two pharmacokinetic models (proposed by Brix and Tofts) that have been applied in the literature. However, a comparison of these models demonstrated that the Brix model is slightly more robust. ${ }^{19}$ Because DCE-MRI has not been established in clinical routine, no definite sequence can be recommended.

Positron emission tomography (PET) in combination with MRI is a novel and promising new methodology in which PET detects active focal lesions, while MRI shows the location of the lesions and provides information on myeloma cell infiltration of the bone marrow. Especially in patients who reach a complete remission (CR), this technique might be able to localize residual sites of disease activity and therefore may help to guide treatment in the future. ${ }^{20}$ 


\section{MRI PATTERNS OF MARROW INVOLVEMENT}

Five MRI patterns of marrow involvement in myeloma have been recognized: normal appearance of bone marrow, focal involvement (positive focal lesion is considered lesion of diameter $\geq 5 \mathrm{~mm}$ ), homogeneous diffuse infiltration, combined diffuse and focal infiltration, and variegated or salt-and-pepper pattern with inhomogeneous bone marrow with interposition of fat islands. ${ }^{21,22}$ Low tumor burden is usually associated with a normal MRI pattern, but a high tumor burden is usually suspected when there is diffuse hypointense change on T1-weighted images, diffuse hyperintensity on T2-weighted images, and enhancement with gadolinium injection. ${ }^{23}$ In several studies, the percentage of symptomatic patients with each of the abnormal MRI bone marrow patterns has ranged from $18 \%$ to $50 \%$ for focal pattern, $25 \%$ to $43 \%$ for diffuse pattern, and $1 \%$ to $5 \%$ for variegated pattern. ${ }^{16}$ The Durie-Salmon PLUS system uses the number of focal lesions (from focal or combined focal and diffuse patterns) for the staging of a patient with myeloma rather than the diffuse or salt-andpepper pattern. ${ }^{24}$

\section{MRI IN SYMPTOMATIC MYELOMA}

\section{MRI Versus Conventional Radiography and Other Imaging Techniques for Detection of Bone Involvement}

MRI is more sensitive compared with WBXR for the detection of bone involvement in MM. In the largest series of patients published to date, MRI was compared with WBXR in 611 patients who received tandem autologous stem-cell transplantation (ASCT). MRI and WBXR detected focal and osteolytic lesions in $74 \%$ and $56 \%$ of the imaged anatomic sites, respectively. Furthermore, $52 \%$ of 267 patients with normal WBXR had focal lesions on MRI. More precisely, MRI detected more focal lesions compared with lytic lesions in WBXR in the spine $(78 \% v 16 \% ; P<.001)$, pelvis $(64 \% v 28 \% ; P<.001)$, and sternum $(24 \% v 3 \% ; P<.001)$. WBXR had better performance than MRI in the ribs $(10 \% v 43 \%$; $P<.001)$ and long bones $(37 \% v 48 \%$; $P=.006$ ) and equal results in the skull and shoulders. ${ }^{25}$ Similar results had been previously reported in smaller studies, where MRI was superior to WBXR for the detection of focal versus osteolytic lesions in the pelvis ( $75 \% v 46 \%$ of patients) and spine (76\% v42\%), especially in the lumbar spine. ${ }^{26-30} \mathrm{~A}$ recent meta-analysis confirmed the superiority of MRI over WBXR regarding the detection of focal lesions and showed that MRI especially outscores WBXR in the axial skeleton but not in the ribs. ${ }^{31}$

Although it is clear that MRI can detect bone marrow focal lesions long before the development of osteolytic lesions on WBXR, other imaging techniques such as PET combined with computed tomography (CT), CT, or WB-CT detect more osteolytic lesions compared with WBXR. ${ }^{31}$ Do we have any evidence that MRI is superior to the other techniques in depicting bone involvement in myeloma? In a study with 41 patients with newly diagnosed MM, WB-MRI was found superior to WB-CT in detecting lesions in the skeleton. ${ }^{32}$ In a prospective study, Zamagni et $\mathrm{al}^{33}$ compared MRI of the spine and pelvis with WBXR versus PET-CT in 46 patients with MM at diagnosis. Although PET-CT was superior to WBXR in detecting lytic lesions in $46 \%$ of patients ( $19 \%$ had negative WBXR), it failed to reveal abnormal findings in 30\% of patients who had abnormal MRI in the same areas, mainly of diffuse pattern. In that study, the combination of spine and pelvic MRI with PET-CT detected both medullary and extramedullary active myeloma sites in almost all patients (92\%). Nevertheless, the Arkansas group was not able to confirm any superiority of MRI over PET-CT in the detection of more focal lesions in a large number of patients $(\mathrm{n}=303)$ within the Total Therapy 3 protocols. ${ }^{34}$ Still, in 188 patients who had $\geq$ one focal lesion on MRI, MRI was superior to PET-CT regarding the detection of higher number of focal lesions $(P=.032)$. Furthermore, in this study, the presence of diffuse marrow pattern was not taken into consideration as an abnormal MRI finding. ${ }^{34}$ Compared with MIBI (sestamibitechnetium99m) scan, WB-MRI detected more lesions in the vertebrae and long bones, produced similar results in the skull, and was inferior in the ribs. ${ }^{35}$ One important question on this point is the value of WBMRI, which is not available everywhere, over MRI of the spine and pelvis. In 100 patients with MM or monoclonal gammopathy of undetermined significance (MGUS) who underwent WB-MRI, $10 \%$ presented with focal lesions merely in the extra-axial skeleton. These lesions would have been ignored if only MRI of the spine and pelvis had been performed. ${ }^{36}$

Other advantages of MRI over WBXR and CT include the discrimination of myeloma from normal marrow ${ }^{4,37}$; this finding can help in the differential diagnosis between myeloma and benign cause of a vertebral fracture. This is of extreme importance in cases of patients with a vertebral fracture and no other CRAB criteria or lytic lesions. MRI can also accurately illustrate the spinal cord and/or nerve root compression for surgical intervention or radiation therapy. ${ }^{2,4}$ Furthermore, the presence of soft tissue extension of MM and presence of extramedullary plasmacytomas, which develop in approximately $10 \%$ to $20 \%$ of patients during the course of their disease, can be precisely visualized by WB-MRI. ${ }^{38-40}$ MRI can also help in the better evaluation of avascular necrosis of the femoral head ${ }^{41}$ and presence of soft tissue amyloid deposits. ${ }^{42}$ Moreover, tumor load can be assessed and monitored by MRI, even in patients with nonsecretory or oligosecretory $\mathrm{MM}^{43}$

\section{Consensus Statement}

MRI is the imaging gold-standard method for the detection of bone marrow involvement in MM (grade A). We stress that MRI detects bone marrow involvement and not bone destruction. MRI of the spine and pelvis can detect approximately $90 \%$ of focal lesions in $\mathrm{MM}$, and thus, it can be used in cases where WB-MRI is not available (grade B).MRI is the procedure of choice to evaluate a painful lesion in patients with myeloma, mainly in the axial skeleton, and detect spinal cord compression (grade A). MRI is particularly useful in the evaluation of collapsed vertebrae, especially when myeloma is not active, where the possibility of osteoporotic fracture is high (grade B).

\section{Prognostic Value of MRI}

The prognostic significance of MRI findings in symptomatic myeloma has been evaluated. The largest study in the literature included 611 patients who received tandem ASCT-based protocols. Focal lesions detected by spinal MRI and not seen on WBXR independently correlated with overall survival (OS). Resolution of the focal lesions on MRI after treatment occurred in $60 \%$ of the patients who had superior survival. At disease progression after CR, MRI revealed new focal lesions in $26 \%$ of patients, enlargement of previous focal lesions in $28 \%$, and both features in $15 \% .{ }^{25}$ In a more recent analysis 
by the same group involving 429 patients, patients who had $>$ seven focal lesions on MRI $(n=147)$ had a $73 \%$ probability of 3-year OS versus $86 \%$ for those who had zero to seven focal lesions $(n=235)$ and $81 \%$ for those who had diffuse pattern of marrow infiltration $(n=47$; $P=.04$ ). PET-CT and WBXR also produced similar results in the univariable analysis. In the multivariable analysis, from the imaging variables, only the presence of $>$ two osteolytic lesions on WBXR at diagnosis and presence of $>$ three focal lesions on PET-CT 7 days after ASCT had independent prognostic value for inferior OS $(P=.01$ and .03 , respectively). However, we have to mention the high percentage of patients (232 [54\%] of 429) who had no detectable osteolytic lesions by WBXR and the absence of evaluation of diffuse MRI pattern in this study. ${ }^{44}$

The MRI pattern of marrow infiltration has also been reported to have prognostic significance in newly diagnosed patients with symptomatic disease..$^{23,45,46}$ In the conventional chemotherapy era, Moulopoulos et $\mathrm{al}^{23}$ reported that the median OS of patients with newly diagnosed MM was 24 months if they had diffuse MRI pattern versus 51, 52, and 56 months for those with focal, variegated, and normal patterns, respectively $(P=.001)$. This is possibly because diffuse MRI marrow pattern correlates with increased angiogenesis and advanced disease features. ${ }^{47,48}$ The same group also reported the prognostic value of MRI patterns in 228 patients with symptomatic MM who received first-line regimens based on novel agents. Patients with diffuse pattern had inferior survival compared with patients with other MRI patterns; moreover, the combination of diffuse MRI pattern, International Staging System stage III, and high-risk cytogenetics could identify a group of patients with poor survival (ie, median, 21 months; probability of 3-year OS, 35\%). ${ }^{46}$ Another study in 126 patients with newly diagnosed symptomatic myeloma who underwent ASCT showed that the diffuse and variegated MRI patterns had an independent predictive value for disease progression (hazard ratio [HR], 1.922; $P=$ $.008) .{ }^{48}$ Finally, in patients with progressive or relapsed MM, an increased DCE-MRI signal indicated shorter progression-free survival, possibly because of its association with higher MVD. ${ }^{15}$

\section{Consensus Statement}

The number of MRI focal lesions ( $>$ seven; grade A) and presence of diffuse pattern (grade B) correlate with inferior survival. Prospective clinical studies are needed to define if these patients have to be treated in a different or more aggressive way.

\section{MRI and Response to Antimyeloma Therapy}

An interesting finding is that a change in MRI pattern correlates with response to therapy. Moulopoulos et $\mathrm{al}^{49}$ first reported in the era of conventional chemotherapy that CR is characterized by complete resolution of the preceding marrow abnormality, whereas partial response is characterized by changeover of diffuse pattern to variegated or focal pattern. In a retrospective study that was conducted in the era of novel agents, response to treatment was compared with changes in infiltration pattern on WB-MRI before and after ASCT $(\mathrm{n}=100)$. There was a strong correlation between response to antimyeloma therapies and changes in both diffuse $(P=.004)$ and focal $(P=.01)$ MRI patterns. Furthermore, the number of focal lesions on second MRI was of prognostic significance for OS $(P=.001) .{ }^{50}$ Another study in 33 patients who underwent ASCT showed that WB-MRI data demonstrated progressive disease in 10 patients (30\%) and response to high-dose therapy in 23 (70\%). Eight (80\%) of the 10 patients with progressive disease revealed intramedullary lesions, and two patients
(20\%) had intra- and extramedullary lesions. WB-MRI had a sensitivity of $64 \%$, specificity of $86 \%$, positive predictive value of $70 \%$, negative predictive value of $83 \%$, and accuracy of $79 \%$ for detection of remission. ${ }^{51}$ This study supports that one of the disadvantages of MRI is that it often provides false-positive results because of persistent nonviable lesions. Thus, PET-CT might be more suitable than MRI for determination of remission status. ${ }^{52}$ Indeed, in a large study of 191 patients, PET-CT revealed faster change of imaging findings than MRI in patients who responded to therapy. ${ }^{53}$ It seems that PET-CT normalization after treatment can offer more information compared with MRI for the better definition of CR. ${ }^{54}$

To improve the results of MRI for the most accurate detection of remission, DW-MRI has been recently used. In a first preliminary report, ADC values in active myeloma were significantly higher than marrow in remission. ${ }^{55}$ Furthermore, the mean ADC increased in 95\% of responding patients and decreased in all nonresponders $(\mathrm{n}=5 ; P=.002)$. An increase of ADC by $3.3 \%$ was associated with response, having a sensitivity of $90 \%$ and specificity of $100 \%$. Furthermore, there was a negative correlation between changes of ADC and changes of biochemical markers of response $(r=-0.614 ; P=.001) .{ }^{56}$ Large prospective clinical studies are definitely justified by these results.

\section{Consensus Statement}

MRI might help in the better definition of CR (grade D; panel consensus). Nevertheless, the high number of false-positive results suggests that its combination with methods that reveal active lesions (ie, PET-MRI) or another imaging method, such as PET-CT, might be of more value in this setting. Thus, the systematic performance of MRI for the follow-up of patients, before or after different therapies, in the absence of clinical indications is not recommended. Novel clinical studies have to include MRI for the response evaluation in an effort to clarify the role of MRI in this important field of myeloma therapy.

\section{VALUE OF MRI IN DEFINITION OF SMM}

The presence of lytic lesions by WBXR is included in the definition of symptomatic myeloma, based on studies showing that patients with $\geq$ one lytic lesion on WBXR have a median time to progression (TTP) of 10 months. ${ }^{57}$ However, in patients with no osteolytic lesions on WBXR, MRI reveals abnormal marrow appearance in $20 \%$ to $50 \%{ }^{22,23,58-60}$; these patients are at higher risk for progression. Moulopoulos et $\mathrm{al}^{58}$ reported that patients with SMM and abnormal MRI studies required therapy after a median of 16 months versus 43 months for those with normal MRI $(P<.01)$. Hillengass et $\mathrm{al}^{59}$ evaluated WB-MRI in 149 patients with SMM. Focal lesions were detected in 42 patients (28\%), while $>$ one focal lesion was present in 23 patients $(15 \%)$ who had high risk of progression (HR, 4.05; $P<$ .001). The median TTP was 13 months, and the progression rate at 2 years was $70 \%$. On multivariable analysis, presence of $>$ one focal lesion remained a significant predictor of progression after adjusting for other risk factors, including bone marrow plasmacytosis, serum and urine $\mathrm{M}$ protein levels, and suppression of uninvolved immunoglobulins. In the same study, diffuse marrow infiltration on MRI was also associated with increased risk for progression (HR, 3.5; $P<.001)$. Kastritis et $\mathrm{al}^{60}$ also showed in a study of 98 patients with SMM that abnormal marrow pattern on MRI of the spine, which was present in 
$21 \%$ of patients, was associated with high risk of progression, with a median TTP to symptomatic myeloma of 15 months $(P=.001)$.

The identification of patients with SMM who are at high risk for progression is of great importance, because these patients may benefit from immediate therapy. A recent randomized study from the Spanish Myeloma Study Group compared the combination of lenalidomide plus low-dose dexamethasone $(\mathrm{Rd})$ versus observation in patients with high-risk SMM (MRI was not used for defining high-risk SMM). TTP was significantly longer with Rd compared with observation (median, not reached $v 21$ months, respectively; $P<.001$ ). More importantly, Rd offered OS advantage (probability of 3-year survival, $94 \%$ v 80\%, respectively; $P=.03) .{ }^{61}$

An important issue is whether patients who have $\geq$ two small focal lesions $(<5 \mathrm{~mm})$ should be considered as having symptomatic myeloma and how to manage them. Recently, the Heidelberg group analyzed data of 63 patients with SMM who had $\geq$ two WB-MRIs performed for follow-up before progression to symptomatic disease. The definition of radiologic progression according to MRI findings included one of the following: development of a new focal lesion, increase of the diameter of an existing focal lesion, or detection of novel or progressive diffuse MRI pattern. The second MRI was performed 3 to 6 months after the performance of the first MRI. Evaluation of response according to IMWG criteria was also performed. Progressive disease according to MRI was observed in approximately $50 \%$ of patients, whereas $40 \%$ of patients developed symptomatic MM based on the CRAB criteria. In the multivariable analysis, progressive disease according to MRI was an independent prognostic factor for progression. Patients with stable MRI findings had no higher risk of progression, even when focal lesions were present on the initial MRI. ${ }^{62}$ Prospective clinical trials should be conducted to confirm these findings.

\section{Consensus Statement}

We recommend that patients with $>$ one unequivocal focal lesion (diameter of $>5 \mathrm{~mm}$ ) should be considered to have symptomatic myeloma that requires therapy (grade B). Patients with equivocal focal lesions should repeat the MRI after 3 to 6 months, and in cases of MRI progression, patients should be considered as symptomatic patients who need therapy (grade C; panel consensus). The biopsy of such lesions should be encouraged. Regarding diffuse MRI marrow pattern, we need additional studies before its incorporation into the definition of symptomatic myeloma.

\section{MRI FINDINGS IN MGUS}

MGUS by definition is characterized by the absence of osteolytic lesions. However, patients with MGUS have higher incidences of osteoporosis and vertebral fractures compared with the normal population. ${ }^{63,64}$ In a small study that included 37 patients with MGUS or SMM, MRI abnormalities were detected in $20 \%$. These patients had a higher TTP to symptomatic myeloma compared with patients with a normal MRI who did not experience progression after a median follow-up of 30 months. ${ }^{65}$ A prospective study involving 331 patients with MGUS or SMM revealed that the detection of multiple ( $>$ one) focal lesions by MRI conferred an increased risk of progression. ${ }^{66}$ In another large study, which included only patients with MGUS ( $\mathrm{n}=$ 137) who underwent WB-MRI at diagnosis, a focal infiltration pattern was detected in $23 \%$. Independent prognostic factors for progression to symptomatic myeloma included the presence and number of focal lesions and value of M-protein. ${ }^{67}$

\section{Consensus Statement}

WB-MRI identifies patients with MGUS with focal lesions that possibly reflect infiltration by monoclonal plasma cells in the bone marrow. These patients seem to have increased risk of progression to myeloma. To date, MRI is not recommended as part of the routine workup for patients with MGUS unless there are clinical features that increase suspicion.

\section{MRI AND SOLITARY BONE PLASMACYTOMA}

The diagnosis of solitary plasmacytoma of the bone (SBP) includes the presence of a solitary bone lesion, with a confirmed infiltration by plasma cells on biopsy of the lesion, absence of clonal plasma cells on the trephine bone marrow biopsy, and no CRAB criteria met. Although definitive radiotherapy usually eradicates the local disease, a majority of patients will develop MM because of the growth of previously occult lesions not detected by WBXR. ${ }^{39}$ Moulopoulos et $\mathrm{al}^{38}$ reported that spinal MRI revealed additional focal lesions in four of 12 patients with SBP. After treatment with radiotherapy to the painful lesion, three patients developed systemic disease within 18 months from diagnosis. Furthermore, Liebross et $\mathrm{al}^{68}$ observed that among patients with SBP with spinal disease, seven of eight staged by WBXR

Table 3. Recommendations for Use of MRI in Myeloma, MGUS, and SBP

\begin{tabular}{|c|c|}
\hline Recommendation & Grade \\
\hline \multicolumn{2}{|l|}{ Symptomatic myeloma } \\
\hline $\begin{array}{l}\mathrm{MRI} \text { is imaging gold-standard method for detection of } \\
\text { bone marrow involvement in } \mathrm{MM}\end{array}$ & A \\
\hline $\begin{array}{l}\text { MRI of spine and pelvis can be used in cases where } \\
\text { WB-MRI is not available }\end{array}$ & B \\
\hline $\begin{array}{l}\mathrm{MRI} \text { is procedure of choice to evaluate painful lesion } \\
\text { and spinal cord compression }\end{array}$ & A \\
\hline $\begin{array}{l}\text { MRI can differentiate malignant from nonmalignant } \\
\text { vertebral fractures }\end{array}$ & B \\
\hline $\begin{array}{l}\text { MRI provides accurate visualization of soft tissue } \\
\text { extension and extramedullary plasmacytoma }\end{array}$ & A \\
\hline Focal pattern provides prognostic information & A \\
\hline Diffuse pattern also correlates with inferior prognosis & $\mathrm{B}$ \\
\hline MRI might help in better definition of CR & D \\
\hline \multicolumn{2}{|l|}{$\mathrm{MRI}$ is not recommended for treatment follow-up } \\
\hline \multicolumn{2}{|l|}{ Asymptomatic myeloma } \\
\hline $\begin{array}{l}\text { Patients with }>\text { one unequivocal focal lesion (diameter } \\
>5 \mathrm{~mm} \text { ) should be considered to have symptomatic } \\
\text { myeloma that requires therapy }\end{array}$ & B \\
\hline $\begin{array}{l}\text { Patients with equivocal focal lesions should repeat MRI } \\
\text { after } 3 \text { to } 6 \text { months; in cases of } \mathrm{MRI} \text { progression, } \\
\text { patients should be considered to be symptomatic } \\
\text { and to require therapy }\end{array}$ & $\mathrm{C}$ \\
\hline \multicolumn{2}{|l|}{ MGUS and SBP } \\
\hline \multicolumn{2}{|l|}{$\begin{array}{l}\text { MRI is not recommended as part of routine workup of } \\
\text { patients with MGUS unless there are clinical } \\
\text { features that increase suspicion }\end{array}$} \\
\hline $\begin{array}{l}\text { MRI should be part of staging procedures in patients } \\
\text { with SBP }\end{array}$ & A \\
\hline
\end{tabular}

Abbreviations: CR, complete response; MGUS, monoclonal gammopathy of undetermined significance; MM, multiple myeloma; MRI, magnetic resonance imaging; SBP, solitary bone plasmacytoma; WB, whole body. 
alone developed MM, compared with only one of seven patients who also underwent spinal MRI.

\section{Consensus Statement}

MRI should be part of the staging procedures in patients with SBP to better assess the extent of the local tumor and reveal occult lesions elsewhere (grade A).

\section{DISCUSSION}

In summary, MRI describes the pattern of myelomatous infiltration of the bone marrow and is the procedure of choice for the evaluation of painful lesions in patients with myeloma for the detection of spinal cord compression and differentiation of malignant from nonmalignant vertebral fractures. MRI provides significant prognostic information in patients with symptomatic disease and may be found useful in the better definition of CR. More importantly, MRI can reclassify patients with SMM to symptomatic MM, and patients with $>$ one unequivocal focal lesion (diameter of $>5 \mathrm{~mm}$ ) should be considered as having symptomatic myeloma that requires therapy. Finally, MRI is part of the staging workup of SBP. A summary of the recommendations is listed in Table 3.
AUTHORS' DISCLOSURES OF POTENTIAL CONFLICTS OF INTEREST

Disclosures provided by the authors are available with this article at www.jco.org.

\section{AUTHOR CONTRIBUTIONS}

Conception and design: Meletios A. Dimopoulos, Evangelos Terpos

Administrative support: Evangelos Terpos

Provision of study materials or patients: Jens Hillengass

Collection and assembly of data: Meletios A. Dimopoulos, Jens

Hillengass, Hartmut Goldschmidt, Pieter Sonneveld, Evangelos Terpos

Data analysis and interpretation: Meletios A. Dimopoulos, Saad Usmani, Elena Zamagni, Suzanne Lentzsch, Faith E. Davies, Noopur Raje, Orhan Sezer, Sonja Zweegman, Jatin Shah, Ashraf Badros, Kazuyuki Shimizu, Philippe Moreau, Chor-Sang Chim, Juan José Lahuerta, Jian Hou, Artur Jurczyszyn, Hartmut Goldschmidt, Pieter Sonneveld, Antonio Palumbo, Heinz Ludwig, Michele Cavo, Bart Barlogie, Kenneth Anderson, G. David Roodman, S. Vincent Rajkumar, Brian G.M. Durie, Evangelos Terpos

Manuscript writing: All authors

Final approval of manuscript: All authors

\section{REFERENCES}

1. International Myeloma Working Group: Criteria for the classification of monoclonal gammopathies, multiple myeloma and related disorders: A report of the International Myeloma Working Group. Br J Haematol 121:749-757, 2003

2. Dimopoulos $\mathrm{M}$, Terpos $\mathrm{E}$, Comenzo RL, et al: International Myeloma Working Group consensus statement and guidelines regarding the current role of imaging techniques in the diagnosis and monitoring of multiple myeloma. Leukemia 23:1545-1556, 2009

3. Durie BG, Salmon SE: A clinical staging system for multiple myeloma: Correlation of measured myeloma cell mass with presenting clinical features, response to treatment, and survival. Cancer 36:842854, 1975

4. Moulopoulos LA, Dimopoulos MA: Magnetic resonance imaging of the bone marrow in hematologic malignancies. Blood 90:2127-2147, 1997

5. Libshitz HI, Malthouse SR, Cunningham D, et al: Multiple myeloma: Appearance at MR imaging. Radiology 182:833-837, 1992

6. Weininger $M$, Lauterbach $B$, Knop $S$, et al: Whole-body MRI of multiple myeloma: Comparison of different MRI sequences in assessment of different growth patterns. Eur J Radiol 69:339-345, 2008

7. Attariwala R, Picker W: Whole body MRI: Improved lesion detection and characterization with diffusion weighted techniques. J Magn Reson Imaging 38:253-268, 2013

8. Müller MF, Edelman RR: Echo planar imaging of the abdomen. Top Magn Reson Imaging 7:112119, 1995

9. Wang $Y$ : Description of parallel imaging in MRI using multiple coils. Magn Reson Med 44:495499, 2000

10. Lemke A, Stieltjes B, Schad LR, et al: Toward an optimal distribution of $B$ values for intravoxel incoherent motion imaging. Magn Reson Imaging 29:766-776, 2011
11. Xu X, Ma L, Zhang JS, et al: Feasibility of whole body diffusion weighted imaging in detecting bone metastasis on 3.0T MR scanner. Chin Med Sci J 23:151-157, 2008

12. Padhani $A R$, Koh DM: Diffusion MR imaging for monitoring of treatment response. Magn Reson Imaging Clin N Am 19:181-209, 2011

13. Messiou C, Collins DJ, Morgan VA, et al: Optimising diffusion weighted MRI for imaging metastatic and myeloma bone disease and assessing reproducibility. Eur Radiol 21:1713-1718, 2011

14. Hillengass J, Bäuerle $T$, Bartl $R$, et al: Diffusion-weighted imaging for non-invasive and quantitative monitoring of bone marrow infiltration in patients with monoclonal plasma cell disease: A comparative study with histology. $\mathrm{Br} J$ Haematol 153:721-728, 2011

15. Hillengass J, Wasser $K$, Delorme $S$, et al: Lumbar bone marrow microcirculation measurements from dynamic contrast-enhanced magnetic resonance imaging is a predictor of event-free survival in progressive multiple myeloma. Clin Cancer Res 13:475-481, 2007

16. Hillengass J, Landgren $\mathrm{O}$ : Challenges and opportunities of novel imaging techniques in monoclonal plasma cell disorders: Imaging "early myeloma." Leuk Lymphoma 54:1355-1363, 2013

17. Huang SY, Chen BB, Lu HY, et al: Correlation among DCE-MRI measurements of bone marrow angiogenesis, microvessel density, and extramedullary disease in patients with multiple myeloma. Am J Hematol 87:837-839, 2012

18. Zechmann $C M$, Traine $L$, Meissner $T$, et al: Parametric histogram analysis of dynamic contrastenhanced MRI in multiple myeloma: A technique to evaluate angiogenic response to therapy? Acad Radiol 19:100-108, 2012

19. Zwick S, Brix G, Tofts PS, et al: Simulationbased comparison of two approaches frequently used for dynamic contrast-enhanced MRI. Eur Radiol 20:432-442, 2010

20. Fraioli F, Punwani S: Clinical and research applications of simultaneous positron emission tomography and MRI. Br J Radiol 87:20130464, 2014
21. Baur-Melnyk $A$, Buhmann $S$, Dürr $H R$, et al: Role of MRI for the diagnosis and prognosis of multiple myeloma. Eur J Radiol 55:56-63, 2005

22. Moulopoulos LA, Varma DG, Dimopoulos MA, et al: Multiple myeloma: Spinal MR imaging in patients with untreated newly diagnosed disease. Radiology 185:833-840, 1992

23. Moulopoulos LA, Gika D, Anagnostopoulos A, et al: Prognostic significance of magnetic resonance imaging of bone marrow in previously untreated patients with multiple myeloma. Ann Oncol 16: 1824-1828, 2005

24. Durie BG: The role of anatomic and functional staging in myeloma: Description of Durie/Salmon plus staging system. Eur J Cancer 42:1539-1543, 2006

25. Walker R, Barlogie B, Haessler J, et al: Magnetic resonance imaging in multiple myeloma: Diagnostic and clinical implications. J Clin Oncol 25: 1121-1128, 2007

26. Ludwig $H$, Frühwald $F$, Tscholakoff $D$, et al: Magnetic resonance imaging of the spine in multiple myeloma. Lancet 2:364-366, 1987

27. Ghanem N, Lohrmann C, Engelhardt M, et al: Whole-body MRI in the detection of bone marrow infiltration in patients with plasma cell neoplasms in comparison to the radiological skeletal survey. Eur Radiol 16:1005-1014, 2006

28. Lecouvet FE, Malghem J, Michaux $L$, et al: Skeletal survey in advanced multiple myeloma: Radiographic versus MR imaging survey. $\mathrm{Br} \mathrm{J}$ Haematol 106:35-39, 1999

29. Tertti $R$, Alanen $A$, Remes $K$ : The value of magnetic resonance imaging in screening myeloma lesions of the lumbar spine. $\mathrm{Br} \mathrm{J}$ Haematol 91:658660,1995

30. Narquin S, Ingrand P, Azais I, et al: Comparison of whole-body diffusion MRI and conventional radiological assessment in the staging of myeloma. Diagn Interv Imaging 94:629-636, 2013

31. Regelink JC, Minnema MC, Terpos E, et al: Comparison of modern and conventional imaging techniques in establishing multiple myeloma-related 
bone disease: A systematic review. $\mathrm{Br} \mathrm{J}$ Haematol 162:50-61, 2013

32. Baur-Melnyk A, Buhmann S, Becker $C$, et al: Whole-body MRI versus whole-body MDCT for staging of multiple myeloma. AJR Am J Roentgenol 190:1097-1104, 2008

33. Zamagni E, Nanni C, Patriarca $F$, et al: $A$ prospective comparison of 18F-fluorodeoxyglucose positron emission tomography-computed tomography, magnetic resonance imaging and whole-body planar radiographs in the assessment of bone disease in newly diagnosed multiple myeloma. Haematologica 92:50-55, 2007

34. Waheed S, Mitchell A, Usmani S, et al: Standard and novel imaging methods for multiple myeloma: Correlates with prognostic laboratory variables including gene expression profiling data. Haematologica 98:71-78, 2013

35. Khalafallah AA, Snarski A, Heng $R$, et al: Assessment of whole body $\mathrm{MRI}$ and sestamibi technetium-99m bone marrow scan in prediction of multiple myeloma disease progression and outcome: A prospective comparative study. BMJ Open 3:pii:e002025, 2013

36. Bäuerle $T$, Hillengass $J$, Fechtner $K$, et al: Multiple myeloma and monoclonal gammopathy of undetermined significance: Importance of wholebody versus spinal MR imaging. Radiology 252:477485, 2009

37. Baur $A$, Stäbler $A$, Brüning $R$, et al: Diffusionweighted MR imaging of bone marrow: Differentiation of benign versus pathologic compression fractures. Radiology 207:349-356, 1998

38. Moulopoulos LA, Dimopoulos MA, Weber D, et al: Magnetic resonance imaging in the staging of solitary plasmacytoma of bone. J Clin Oncol 11: 1311-1315, 1993

39. Dimopoulos MA, Moulopoulos LA, Maniatis A, et al: Solitary plasmacytoma of bone and asymptomatic multiple myeloma. Blood 96:2037-2044, 2000

40. Varettoni M, Corso A, Pica G, et al: Incidence, presenting features and outcome of extramedullary disease in multiple myeloma: A longitudinal study on 1003 consecutive patients. Ann Oncol 21:325-330, 2010

41. Lafforgue $P$, Dahan $E$, Chagnaud $C$, et al: Early-stage avascular necrosis of the femoral head: MR imaging for prognosis in 31 cases with at least 2 years of follow-up. Radiology 187:199-204, 1993

42. Syed IS, Glockner JF, Feng D, et al: Role of cardiac magnetic resonance imaging in the detection of cardiac amyloidosis. JACC Cardiovasc Imaging 3:155-164, 2010

43. Carlson K, Aström G, Nyman R, et al: MR imaging of multiple myeloma in tumour mass measurement at diagnosis and during treatment. Acta Radiol 36:9-14, 1995
44. Usmani SZ, Mitchell A, Waheed S, et al: Prognostic implications of serial 18-fluoro-deoxyglucose emission tomography in multiple myeloma treated with total therapy. Blood 121:1819-1823, 2013

45. Lecouvet FE, Vande Berg BC, Michaux $L$, et al: Stage III multiple myeloma: Clinical and prognostic value of spinal bone marrow MR imaging. Radiology 209:653-660, 1998

46. Moulopoulos LA, Dimopoulos MA, Kastritis E, et al: Diffuse pattern of bone marrow involvement on magnetic resonance imaging is associated with high risk cytogenetics and poor outcome in newly diagnosed, symptomatic patients with multiple myeloma: A single center experience on 228 patients. Am J Hematol 87:861-864, 2012

47. Moulopoulos LA, Dimopoulos MA, Christoulas D, et al: Diffuse MRI marrow pattern correlates with increased angiogenesis, advanced disease features and poor prognosis in newly diagnosed myeloma treated with novel agents. Leukemia 24: 1206-1212, 2010

48. Song MK, Chung JS, Lee JJ, et al: Magnetic resonance imaging pattern of bone marrow involvement as a new predictive parameter of disease progression in newly diagnosed patients with multiple myeloma eligible for autologous stem cell transplantation. Br J Haematol 165:777-785, 2014

49. Moulopoulos LA, Dimopoulos MA, Alexanian $\mathrm{R}$, et al: Multiple myeloma: MR patterns of response to treatment. Radiology 193:441-446, 1994

50. Hillengass J, Ayyaz S, Kilk K, et al: Changes in magnetic resonance imaging before and after autologous stem cell transplantation correlate with response and survival in multiple myeloma. Haematologica 97: 1757-1760, 2012

51. Bannas $P$, Hentschel HB, Bley TA, et al: Diagnostic performance of whole-body MRI for the detection of persistent or relapsing disease in multiple myeloma after stem cell transplantation. Eur Radiol 22:2007-2012, 2012

52. Derlin T, Peldschus K, Münster $S$, et al: Comparative diagnostic performance of ${ }^{18} \mathrm{~F}-\mathrm{FDG}$ PET/CT versus whole-body MRI for determination of remission status in multiple myeloma after stem cell transplantation. Eur Radiol 23:570-578, 2013

53. Spinnato $P$, Bazzocchi $A$, Brioli $A$, et al: Contrast enhanced $\mathrm{MRI}$ and ${ }^{18} \mathrm{~F}-\mathrm{FDG}$ PET-CT in the assessment of multiple myeloma: A comparison of results in different phases of the disease. Eur $J$ Radiol 81:4013-4018, 2012

54. Bartel TB, Haessler J, Brown TL, et al: F18fluorodeoxyglucose positron emission tomography in the context of other imaging techniques and prognostic factors in multiple myeloma. Blood 114 : 2068-2076, 2009

55. Messiou C, Giles S, Collins DJ, et al: Assessing response of myeloma bone disease with diffusion-weighted MRI. Br J Radiol 85:e1198e1203, 2012
56. Giles SL, Messiou C, Collins DJ, et al: Wholebody diffusion-weighted MR imaging for assessment of treatment response in myeloma. Radiology 271:785-794, 2014

57. Dimopoulos MA, Moulopoulos A, Smith T, et al: Risk of disease progression in asymptomatic multiple myeloma. Am J Med 94:57-61, 1993

58. Moulopoulos LA, Dimopoulos MA, Smith TL, et al: Prognostic significance of magnetic resonance imaging in patients with asymptomatic multiple myeloma. J Clin Oncol 13:251-256, 1995

59. Hillengass J, Fechtner K, Weber MA, et al: Prognostic significance of focal lesions in wholebody magnetic resonance imaging in patients with asymptomatic multiple myeloma. J Clin Oncol 28: 1606-1610, 2010

60. Kastritis E, Terpos E, Moulopoulos L, et al: Extensive bone marrow infiltration and abnormal free light chain ratio identifies patients with asymptomatic myeloma at high risk for progression to symptomatic disease. Leukemia 27:947-953, 2013

61. Mateos MV, Hernández MT, Giraldo $P$, et al: Lenalidomide plus dexamethasone for high-risk smoldering multiple myeloma. N Engl J Med 369: 438-447, 2013

62. Merz M, Hielscher T, Wagner B, et al: Predictive value of longitudinal whole-body magnetic resonance imaging in patients with smoldering multiple myeloma. Leukemia 28:1902-1908, 2014

63. Pepe J, Petrucci MT, Nofroni I, et al: Lumbar bone mineral density as the major factor determining increased prevalence of vertebral fractures in monoclonal gammopathy of undetermined significance. Br J Haematol 134:485-490, 2006

64. van de Donk NW, Palumbo A, Johnsen $\mathrm{HE}_{\text {, et }}$ al: The clinical relevance and management of monoclonal gammopathy of undetermined significance and related disorders: Recommendations from the European Myeloma Network. Haematologica 99: 984-996, 2014

65. Vande Berg BC, Michaux L, Lecouvet FE, et al: Nonmyelomatous monoclonal gammopathy: Correlation of bone marrow MR images with laboratory findings and spontaneous clinical outcome. Radiology 202:247-251, 1997

66. Dhodapkar MV, Sexton R, Waheed S, et al: Clinical, genomic, and imaging predictors of myeloma progression from asymptomatic monoclonal gammopathies (SWOG S0120). Blood 123:78-85, 2014

67. Hillengass J, Weber MA, Kilk K, et al: Prognostic significance of whole-body $\mathrm{MRI}$ in patients with monoclonal gammopathy of undetermined significance. Leukemia 28:174-178, 2014

68. Liebross $\mathrm{RH}, \mathrm{Ha}$ CS, Cox JD, et al: Solitary bone plasmacytoma: Outcome and prognostic factors following radiotherapy. Int J Radiat Oncol Biol Phys 41:1063-1067, 1998

Meletios A. Dimopoulos and Evangelos Terpos, National and Kapodistrian University of Athens School of Medicine, Athens, Greece; Jens Hillengass and Hartmut Goldschmidt, University Hospital Heidelberg, Heidelberg, Germany; Saad Usmani, Carolinas Healthcare System, Charlotte, NC; Elena Zamagni and Michele Cavo, Bologna University School of Medicine, Bologna; Antonio Palumbo, S. Giovanni Battista Hospital, University of Turin, Turin, Italy; Suzanne Lentzsch, Columbia University College of Physicians and Surgeons, New York, NY; Faith E. Davies, Institute of Cancer Research, Sutton, United Kingdom; Noopur Raje, Massachusetts General Hospital Cancer Center; Kenneth Anderson, Dana-Farber Brigham and Women's Cancer Center, Harvard Medical School, Boston, MA; Orhan Sezer, Memorial Sisli Hospital, Istanbul, Turkey; Sonja Zweegman, VU University Medical Center, Amsterdam; Pieter Sonneveld, Erasmus University Medical Center, Rotterdam, the Netherlands; Jatin Shah, MD Anderson Cancer Center, Houston, TX; Ashraf Badros, University of Maryland Medical Center, Baltimore, MD; Kazuyuki Shimizu, Tokai Central Hospital, Kakamigahara, Japan; Philippe Moreau, University Hospital Hôtel-Dieu, 
Nantes, France; Chor-Sang Chim, Queen Mary Hospital, University of Hong Kong, Hong Kong, Special Administrative Region; Jian Hou, Changzheng Hospital, Second Military Medical University, Shanghai, People’s Republic of China; Juan José Lahuerta, Hospital Universitario 12 de Octubre, Madrid, Spain; Artur Jurczyszyn, University Hospital, Krakow, Poland; Heinz Ludwig, Wilhelminenspital, Vienna, Austria; Bart Barlogie, University of Arkansas for Medical Sciences, Little Rock, AR; G. David Roodman, Indiana University School of Medicine, Indianapolis, IN; S. Vincent Rajkumar, Mayo Clinic, Rochester, MN; and Brian G.M. Durie, Samuel Oschin Comprehensive Cancer Institute, Los Angeles, CA.

\section{GLOSSARY TERMS}

dynamic contrast-enhanced magnetic resonance imaging (DCE-MRI): a magnetic resonance imaging acquisition strategy involving multiple scans over a set volume during injection of a magnetic resonance contrast agent. microvessel density (MVD): a quantification technique used to assess the number of vessels in a particular tumor specimen using immunohistochemical stains for endothelial markers. High MVD has been found to be associated with poor prognosis in patients with solid tumors.

\section{Not an ASCO Member? Subscribe to Journal of Oncology Practice}

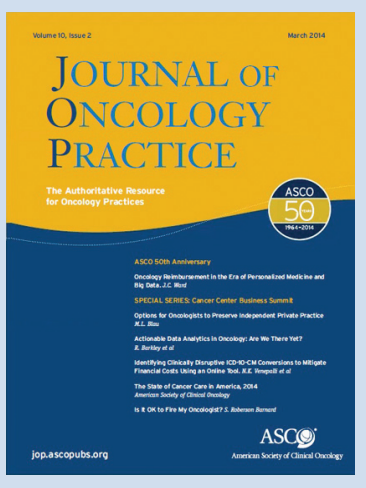

Journal of Oncology Practice (JOP) is ASCO's bimonthly forum for providing its subscribers with information, news, and tools to enhance practice efficiency and promote a high standard of quality for patient care.

Every issue of $J O P$ includes important features on cancer policy issues and their practical effect on cancer care, methods for enhancing the quality of patient care, and tools for improving practice management.

Whether practitioners are in an office or hospital setting, a community or academic environment, JOP provides practical information and advice that oncologists and other oncology professionals can apply immediately to their practices. Key features include:

- Articles for all members of the practice-physicians, nurses, and administrators

- Timely and relevant information to help practices succeed

- Focus on improving practice efficiency and quality of care

- Coverage of legal, financial, technology, and personnel issues

Subscribe today at jop.ascopubs.org. 


\section{AUTHORS' DISCLOSURES OF POTENTIAL CONFLICTS OF INTEREST}

Role of Magnetic Resonance Imaging in the Management of Patients With Multiple Myeloma: A Consensus Statement

The following represents disclosure information provided by authors of this manuscript. All relationships are considered compensated. Relationships are self-held unless noted. I = Immediate Family Member, Inst = My Institution. Relationships may not relate to the subject matter of this manuscript. For more information about ASCO's conflict of interest policy, please refer to www.asco.org/rwc or jco.ascopubs.org/site/ifc.

Meletios A. Dimopoulos

Consulting or Advisory Role: Ortho Biotech (Inst), Celgene (Inst), Onyx Pharmaceuticals (Inst)

\section{Jens Hillengass}

Honoraria: Celgene, Janssen-Cilag, Medtronic

Travel, Accommodations, Expenses: Celgene, Janssen-Cilag, Medtronic

\section{Saad Usmani}

Honoraria: Celgene, Onyx Pharmaceuticals, sanofi-aventis, Millennium Pharmaceuticals

Consulting or Advisory Role: Celgene, Onyx Pharmaceuticals, sanofi-aventis, Millennium Pharmaceuticals

Speakers' Bureau: Celgene, Onyx Pharmaceuticals, Millennium

Pharmaceuticals

Research Funding: Celgene, Onyx Pharmaceuticals, Janssen-Cilag, Array BioPharma, Pharmacyclics

\section{Elena Zamagni}

No relationship to disclose

\section{Suzanne Lentzsch}

Consulting or Advisory Role: Celgene, Bristol-Myers Squibb, Millennium Pharmaceuticals, Janssen

Speakers' Bureau: Axiom

Research Funding: Celgene

Faith E. Davies

Honoraria: Celgene, Janssen-Cilag

Consulting or Advisory Role: Millennium Pharmaceuticals, Onyx

Pharmaceuticals, Celgene, Janssen-Cilag

\section{Noopur Raje}

Consulting or Advisory Role: Celgene, Millennium Pharmaceuticals, Amgen, Novartis

Research Funding: Eli Lilly, Acetylon Pharmaceuticals

Orhan Sezer

Honoraria: Celgene, Janssen-Cilag

Consulting or Advisory Role: Celgene, Janssen-Cilag

\section{Sonja Zweegman}

Consulting or Advisory Role: Celgene (Inst), Janssen-Cilag (Inst), Millennium Pharmaceuticals (Inst)

Research Funding: Celgene (Inst), Janssen-Cilag (Inst), Millennium Pharmaceuticals (Inst)

\section{Jatin Shah}

Consulting or Advisory Role: Celgene, Novartis, Millennium

Pharmaceuticals, Onyx Pharmaceuticals, sanofi-aventis, Array

BioPharma

Research Funding: Celgene, Millennium Pharmaceuticals, Novartis, Onyx Pharmaceuticals, Array BioPharma

\section{Ashraf Badros}

No relationship to disclose

\section{Kazuyuki Shimizu}

Consulting or Advisory Role: Daiichi Sankyo

Expert Testimony: Amgen

Philippe Moreau

No relationship to disclose

\section{Chor-Sang Chim}

Honoraria: Janssen Oncology, Novartis

Consulting or Advisory Role: Novartis

Speakers' Bureau: Janssen Oncology

Travel, Accommodations, Expenses: Janssen Oncology, Novartis

Juan José Lahuerta

Consulting or Advisory Role: Celgene, Janssen-Cilag

Travel, Accommodations, Expenses: Celgene

\section{Jian Hou}

No relationship to disclose

Artur Jurczyszyn

Travel, Accommodations, Expenses: Celgene, Teva

Hartmut Goldschmidt

Honoraria: Janssen Pharmaceuticals, Celgene, Novartis, Chugai

Pharmaceutical, Onyx Pharmaceuticals, Millennium Pharmaceuticals

Consulting or Advisory Role: Janssen Pharmaceuticals (Inst), Celgene

(Inst), Novartis (Inst), Onyx Pharmaceuticals (Inst), Millennium

Pharmaceuticals (Inst)

Research Funding: Janssen Pharmaceuticals (Inst), Celgene (Inst), Novartis (Inst), Chugai Pharmaceutical (Inst)

\section{Pieter Sonneveld}

Consulting or Advisory Role: Celgene (Inst), Onyx Pharmaceuticals (Inst), Janssen-Cilag (Inst), Millennium Pharmaceuticals (Inst), Novartis Research Funding: Celgene (Inst), Onyx Pharmaceuticals (Inst), Janssen-Cilag (Inst)

\section{Antonio Palumbo}

Honoraria: Amgen, Bristol-Myers Squibb, Array BioPharma, Genmab, Celgene, Janssen-Cilag, Millennium Pharmaceuticals, BiPar/ sanofi-aventis, Onyx Pharmaceuticals

Consulting or Advisory Role: Bristol-Myers Squibb, Amgen, Genmab, Celgene, Janssen-Cilag, Millennium Pharmaceuticals, Onyx

Pharmaceuticals

\section{Heinz Ludwig}

Consulting or Advisory Role: Celgene (Inst), Janssen-Cilag (Inst), Onyx Pharmaceuticals (Inst), Bristol-Myers Squibb (Inst)

Speakers' Bureau: Celgene, Janssen-Cilag

Research Funding: Takeda Pharmaceuticals (Inst)

Travel, Accommodations, Expenses: Sandoz

\section{Michele Cavo}

Honoraria: Janssen-Cilag, Celgene, Millennium Pharmaceuticals, Onyx Pharmaceuticals, Bristol-Myers Squibb

Consulting or Advisory Role: Janssen-Cilag, Celgene, Millennium

Pharmaceuticals

Speakers' Bureau: Janssen-Cilag, Celgene

\section{Bart Barlogie}

Consulting or Advisory Role: Celgene, Millennium Pharmaceuticals Research Funding: Celgene

Patents, Royalties, Other Intellectual Property: Myeloma Health

Travel, Accommodations, Expenses: Celgene, Multiple Myeloma Research Foundation, Dana-Farber Cancer Institute

Kenneth Anderson

No relationship to disclose 


\section{G. David Roodman}

Consulting or Advisory Role: Amgen

Research Funding: Eli Lilly (Inst)

\section{S. Vincent Rajkumar}

No relationship to disclose

Brian G.M. Durie

No relationship to disclose

\section{Evangelos Terpos}

Honoraria: Celgene, Medtronic, Onyx Pharmaceuticals, Novartis, Janssen-Cilag, Amgen

Consulting or Advisory Role: Roche (Inst), Teva (Inst), Acetylon Pharmaceuticals (Inst), Amgen (Inst), Celgene (Inst)

Research Funding: Amgen (Inst), Janssen-Cilag (Inst)

Travel, Accommodations, Expenses: Janssen-Cilag, Genesis 


\section{Appendix}

Members of the International Myeloma Working Group: Niels Abildgaard, Syddansk Universitet, Odense, Denmark; Rafat Abonour, Indiana University School of Medicine, Indianapolis, IN; Melissa Alsina, H. Lee Moffitt Cancer Center and Research Institute, Tampa, FL; Kenneth C. Anderson, Dana-Farber Cancer Institute, Boston, MA; Michel Attal, Purpan Hospital, Toulouse, France; Hervé Avet-Loiseau, University of Toulouse, Toulouse, France; Ashraf Badros, University of Maryland, Baltimore, MD; Bart Barlogie, Myeloma Institute for Research and Therapy, University of Arkansas for Medical Sciences, Little Rock, AR; Régis Bataille, Institute de Biologie, Nantes, France; Meral Beksaç, Ankara University, Ankara, Turkey; Andrew Belch, University of Alberta, Edmonton, Alberta, Canada; Dina Ben-Yehuda, Hadassah University Hospital, Hadassah, Israel; Bill Bensinger, Fred Hutchinson Cancer Center, Seattle, WA; P. Leif Bergsagel, Mayo Clinic Scottsdale, Scottsdale, AZ; Joan Bladé, Hospital Clinica, Barcelona, Spain; Mario Boccadoro, University of Torino, Torino, Italy; Jo Caers, Centre Hospitalier Universitaire de Liège, Liège, Belgium; Michele Cavo, Universita di Bologna, Bologna, Italy; Asher ChananKhan, Mayo Clinic, Jacksonville, FL; Wen Ming Chen, Beijing Chaoyang Hospital, Beijing, People's Republic of China; Marta Chesi, Mayo Clinic Scottsdale, Scottsdale, AZ; J. Anthony Child, Leeds General Hospital, Leeds, United Kingdom; Chor Sang Chim, Queen Mary Hospital, Hong Kong, Special Administrative Region, People's Republic of China; Wee-Joo Chng, National University Health System, Singapore, Singapore; Ray Comenzo, Tufts Medical School, Boston, MA; John Crowley, Cancer Research and Biostatistics, Seattle, WA; William Dalton, H. Lee Moffitt, Tampa, FL; Faith E. Davies, Royal Marsden Hospital, London, United Kingdom; Javier de la Rubia, Hospital Universitario La Fe, Valencia, Spain; Cármino de Souza, Univeridade de Campinas, Caminas, Brazil; Michel Delforge, University Hospital Gasthuisberg, Leuven, Belgium; Meletios A. Dimopoulos, University of Athens School of Medicine, Athens, Greece; Angela Dispenzieri, Mayo Clinic, Rochester, MN; Johannes Drach, University of Vienna, Vienna, Austria; Matthew Drake, Mayo Clinic, Rochester, MN; Juan Du, Changzhen Hospital, Shanghai, People’s Republic of China; Brian G.M. Durie, Cedars-Sinai Samuel Oschin Cancer Center, Los Angeles, CA; Hermann Einsele, Universitätsklinik Würzburg, Würzburg, Germany; Thierry Facon, Centre Hospitalier Regional Universitaire de Lille, Lille, France; Dorotea Fantl, Socieded Argentinade Hematolgia, Buenos Aires, Argentina; Jean-Paul Fermand, Hopital Saint-Louis, Paris, France; Carlos Fernández de Larrea, Hospital Clínic de Barcelona, Barcelona, Spain; Rafael Fonseca, Mayo Clinic Scottsdale, Scottsdale, AZ; Gösta Gahrton, Karolinska Institute for Medicine, Huddinge, Sweden; Ramón García-Sanz, University Hospital of Salamanca, Salamanca, Spain; Laurent Garderet, Hopital Saint Antoine, Paris, France; Christina Gasparetto, Duke University Medical Center, Durham, NC; Morie Gertz, Mayo Clinic, Rochester, MN; Irene Ghobrial, Dana-Farber Cancer Institute, Boston, MA; John Gibson, Royal Prince Alfred Hospital, Sydney, New South Wales, Australia; Peter Gimsing, University of Copenhagen, Copenhagen, Denmark; Sergio Giralt, Memorial Sloan-Kettering Cancer Center, New York, NY; Hartmut Goldschmidt, University Hospital Heidelberg, Heidelberg, Germany; Jingli Gu, The First Hospital, Sun Yat-Sen University, Guangdong, People's Republic of China; Roman Hajek, University Hospital Ostrava and University of Ostrava School of Medicine, Ostrava, Czech Republic; Izhar Hardan, Tel Aviv University, Tel Aviv, Israel; Parameswaran Hari, Medical College of Wisconsin, Milwaukee, WI; Hiroyuki Hata, Kumamoto University Hospital, Kumamoto, Japan; Yutaka Hattori, Keio University School of Medicine, Tokyo, Japan; Tom Heffner, Emory University, Atlanta, GA; Jens Hillengass, University of Heidelberg, Heidelberg, Germany; Joy Ho, Royal Prince Alfred Hospital, Sydney, New South Wales, Australia; Antje Hoering, Cancer Research and Biostatistics, Seattle, WA; Jian Hou, Shanghai Chang Zheng Hospital, Shanghai, People's Republic of China; Jeffrey Huang, National Taiwan University Hospital, Taipei, Taiwan; Vania Hungria, Clinica San Germano, Sao Paolo, Brazil; Shinsuke Ida, Nagoya City University Medical School, Nagoya, Japan; Peter Jacobs, Constantiaberg Medi-Clinic, Plumstead, South Africa; Sundar Jagannath, Mt. Sinai Cancer Institute, New York, NY; Andrzej J. Jakubowiak, University of Chicago, Chicago, IL; Hans Johnsen, Aalborg Hospital Science and Innovation Center, Aalborg, Denmark; Douglas Joshua, Royal Prince Alfred Hospital, Sydney, New South Wales, Australia; Artur Jurczyszyn, University Hospital, Krakow, Poland; Efstathios Kastritis, University of Athens, Athens, Greece; Jonathan Kaufman, Emory Clinic, Atlanta, GA; Michio Kawano, Yamaguchi University, Ube, Japan; Neha Korde, National Institutes of Health, Bethesda, MD; Eva Kovacs, Cancer Immunology Research-Life, Birsfelden, Switzerland; Amrita Krishnan, City of Hope, Duarte, CA; Sigurdur Kristinsson, Karolinska University Hospital and Karolinska Institutet, Stockholm, Sweden; Nicolaus Kröger, University Hospital Hamburg, Hamburg, Germany; Shaji Kumar, Mayo Clinic, Rochester, MN; Robert A. Kyle, Mayo Clinic, Rochester, MN; Chara Kyriacou, Northwick Park Hospital, London, United Kingdom; Martha Lacy, Mayo Clinic, Rochester, MN; Juan José Lahuerta, Grupo Español di Mieloma, Hospital Universitario 12 de Octubre, Madrid, Spain; Ola Landgren, Memorial Sloan-Kettering Cancer Center, New York, NY; Alessandra LaRocca, Divisione Universitaria di Ematologia, Torino, Italy; Jacob Laubach, Dana-Farber Cancer Institute, Boston, MA; Fernando Leal da Costa, Instituto Portugues De Oncologia, Lisbon, Portugal; Jae-Hoon Lee, Gachon University Gil Hospital, Incheon, Korea; Merav Leiba, Sheba Medical Center, Tel Hashomer, Israel; Xavier LeLeu, Hospital Huriez, Centre Hospitalier R[actue] egional Universitaire, Lille, France; Suzanne Lentzsch, Columbia University, New York, NY; Nelson Leung, Mayo Clinic, Rochester, MN; Henk Lokhorst, University Medical Center Utrecht, Utrecht, the Netherlands; Sagar Lonial, Emory University Medical School, Atlanta, GA; Jin Lu, People's Hospital, Beijing University, Beijing, People's Republic of China; Heinz Ludwig, Wilhelminenspital der Stat Wien, Vienna, Austria; Anuj Mahindra, University of California at San Francisco, San Francisco, CA; Angelo Maiolino, Rua fonte da Saudade, Rio de Janeiro, Brazil; María-Marivi Mateos, University Hospital of Salamanca-Instituto de Investigación Biomédica de Salamanca (IBSAL), Instituto de Biología Molecular y Celular del Cancer (IBMCC; Universidad de Salamanca [USAL] -Centro Superior de Investigaciones Científicas [CSIC]), Salamanca, Spain; Amitabha Mazumder, New York University Comprehensive Cancer Center, New York, NY; Philip McCarthy, Roswell Park Cancer Center, Buffalo, NY; Jayesh Mehta, Northwestern 
University, Chicago, IL; Ulf-Henrik Mellqvist, Sahlgrenska University Hospital, Gothenburg, Sweden; Giampaolo Merlini, University of Pavia, Pavia, Italy; Joseph Mikhael, Mayo Clinic Scottsdale, Scottsdale, AZ; Philippe Moreau, University Hospital, Nantes, France; Gareth Morgan, Royal Marsden Hospital, London, United Kingdom; Nikhil Munshi, Dana-Farber Cancer Institute, Boston, MA; Hareth Nahi, Karolinska University Hospital, Stockholm, Sweden; Weerasak Nawarawong, Chiang Mai University, Chiang Mai, Thailand; Ruben Niesvizky, Weill Cornell Medical College, New York, NY; Amara Nouel, Hospital Rutz y Paez, Bolivar, Venezuela; Yana Novis, Hospital Sírio Libanês, Bela Vista, Brazil; Enrique Ocio, University Hospital of Salamanca-IBSAL, IBMCC (USAL-CSIC), Salamanca, Spain; Alberto Orfao, University Hospital of Salamanca-IBSAL, IBMCC (USAL-CSIC), Salamanca, Spain; Robert Orlowski, MD Anderson Cancer Center, Houston, TX; Bruno Paiva, Clinica Universitaria de Navarra, Centro de Investigación Médica Aplicada (CIMA), Pamplona, Spain; Antonio Palumbo, University of Torino, Torino, Italy; Santiago Pavlovsky, Fundaleu, Buenos Aires, Argentina; Linda Pilarski, University of Alberta, Edmonton, Alberta, Canada; Raymond Powles, Parkside Cancer Centre, London, United Kingdom; Noopur Raje, Massachusetts General Hospital, Boston, MA; S. Vincent Rajkumar, Mayo Clinic, Rochester, MN; Donna Reece, Princess Margaret Hospital, Toronto, Ontario, Canada; Anthony Reiman, Saint John Regional Hospital, Saint John, New Brunswick, Canada; Paul G. Richardson, Dana-Farber Cancer Institute, Boston, MA; Angelina Rodríguez Morales, Bonco Metro Politano de Sangre, Caracas, Venezuela; Kenneth R. Romeril, Wellington Hospital, Wellington, New Zealand; David Roodman, Indiana University, Indianapolis, IN; Laura Rosiñol, Hospital Clinic, Barcelona, Spain; Murielle Rousseau, University of Toulouse, Toulouse, France; Stephen Russell, Mayo Clinic, Rochester, MN; Jesús San Miguel, Clinica Universitaria de Navarra, CIMA, Pamplona, Spain; Rik Schots, Universitair Ziekenhuis Brussel, Brussels, Belgium; Sabina Sevcikova, Masaryk University, Brno, Czech Republic; Orhan Sezer, Memorial Sisli Hastanesi, Istanbul, Turkey; Jatin J. Shah, MD Anderson Cancer Institute, Houston, TX; Kazuyuki Shimizu, Tokai Central Hospital, Kakamigahara, Japan; Chaim Shustik, McGill University, Montreal, Quebec, Canada; David Siegel, Hackensack University Medical Center, Hackensack, NJ; Seema Singhal, Northwestern University, Chicago, IL; Pieter Sonneveld, Erasmus University Medical Center, Rotterdam, the Netherlands; Andrew Spencer, Alfred Hospital, Melbourne, Victoria, Australia; Edward Stadtmauer, University of Pennsylvania, Philadelphia, PA; Keith Stewart, Mayo Clinic Scottsdale, Scottsdale, AZ; Daryl Tan, Singapore General Hospital, Singapore, Singapore; Evangelos Terpos, University of Athens School of Medicine, Athens, Greece; Patrizia Tosi, Italian Cooperative Group, Istituto di Ematologia Seragnoli, Bologna, Italy; Guido Tricot, University of Iowa Hospital and Clinics, Iowa City, IA; Ingemar Turesson, Skane University Hospital, Malmo, Sweden; Saad Usmani, Levine Cancer Institute/Carolinas Healthcare System, Charlotte, NC; Ben Van Camp, Vrije Universiteit Brussel, Brussels, Belgium; Brian Van Ness, University of Minnesota, Minneapolis, MN; Ivan Van Riet, Vrije Universiteit Brussel (VUB), Brussels, Belgium; Isabelle Vande Broek, VUB, Brussels, Belgium; Karin Vanderkerken, VUB, Brussels, Belgium; Robert Vescio, Cedars-Sinai Cancer Center, Los Angeles, CA; David Vesole, Hackensack University Medical Center, Hackensack, NJ; Peter Voorhees, University of North Carolina, Chapel Hill, NC; Anders Waage, University Hospital, Trondheim, Norway; Michael Wang, MD Anderson Cancer Center, Houston, TX; Donna Weber, MD Anderson Cancer Center, Houston, TX; Jan Westin, Sahlgrenska University Hospital, Gothenburg, Sweden; Keith Wheatley, University of Birmingham, Birmingham, United Kingdom; Elena Zamagni, University of Bologna, Bologna, Italy; Jeffrey Zonder, Karmanos Cancer Institute, Detroit, MI; and Sonja Zweegman, VU University Medical Center, Amsterdam, the Netherlands. 